\title{
Sentinel lymph node identification in early stage ovarian cancer: is it still possible after prior tumor resection?
}

\author{
Pim Laven ${ }^{1,2^{*}} \mathbb{C}$, Roy Kruitwagen ${ }^{1,2}$, Petra Zusterzeel ${ }^{3}$, Brigitte Slangen ${ }^{1,2}$, Toon van Gorp ${ }^{1,2,4}$, \\ Jochem van der Pol ${ }^{5}$ and Sandrina Lambrechts ${ }^{1,2}$
}

\begin{abstract}
Objective: Sentinel lymph node (SLN) detection in ovarian cancer is feasible when tracers are injected before the pathological ovary is resected. This study aims to investigate whether the SLN identification is also feasible in patients whose ovarian tumor has already been resected with injection of the tracer into the ovarian ligaments stumps, i.e. in the event that a frozen section confirms malignancy.

Methods: Patients who underwent laparotomy with frozen section confirming an ovarian malignancy, and those who underwent a second staging laparotomy after prior resection of a malignant ovarian mass, were included. Blue dye and a radioactive isotope were injected in the stumps of the ligamentum ovarium proprium and the ligamentum infundibulo-pelvicum. After an interval of at least 15-min, the sentinel node(s) were identified using either the gamma-probe and / or blue dye.
\end{abstract}

Results: A total of 11 patients were included in the study, the sentinel node (SLN) procedure was completed in all 11 patients. At least one SLN was identified in 3 patients, resulting in a rather low detection rate of $27,3 \%$.

Conclusion: In this study we showed that SLN procedure after (previous) resection of the tumor seems inferior to detect sentinel nodes when compared to injection of the tracer in the ovarian ligaments before tumor resection.

Trial registration: NCT02540551

Keywords: Ovarian cancer, Sentinel lymph node

\section{Introduction}

The International Federation of Gynecology and Obstetrics recommends surgical staging in patients with clinical early-stage epithelial ovarian cancer (EOC) including pelvic and para-aortic lymphadenectomy to detect lymph node metastases. There is 14\% (range 6.1-29.6\%) chance of finding lymph node metastases, implicating pathological advanced stage disease with an indication for adjuvant

*Correspondence: pim.laven@mumc.nl

${ }^{2}$ GROW - School for Oncology and Developmental Biology, Maastricht University Medical Center, Maastricht, The Netherlands

Full list of author information is available at the end of the article chemotherapy [1]. The more lymph nodes are removed, the higher the likelihood of detecting metastases [2]. Although complete pelvic and para-aortic lymphadenectomy has been shown to detect up to 250 lymph nodes, radical lymphadenectomy has been associated with serious potential morbidity [3-5] and there is therefore a great difference in the extent of lymph node dissection between different centers [6-8].

The concept of sentinel lymph node (SLN) surgery is widely used in different tumor types to assess whether the cancer has spread to the first lymph node in the lymph drainage pathway, called the SLN. The absence of cancer cells in the SLN is associated with a high probability that original author(s) and the source, provide a link to the Creative Commons licence, and indicate if changes were made. The images or other third party material in this article are included in the article's Creative Commons licence, unless indicated otherwise in a credit line to the material. If material is not included in the article's Creative Commons licence and your intended use is not permitted by statutory regulation or exceeds the permitted use, you will need to obtain permission directly from the copyright holder. To view a copy of this licence, visit http://creativecommons.org/licenses/by/4.0/. The Creative Commons Public Domain Dedication waiver (http://creativeco mmons.org/publicdomain/zero/1.0/) applies to the data made available in this article, unless otherwise stated in a credit line to the data. 
the cancer has not spread to other lymph nodes. The SLN technique has proven effective in the staging of various types of cancer, including breast cancer, malignant melanoma, and vulvar cancer [9-11]. In these tumor types the SLN technique has replaced the systematic performance of complete lymphadenectomy, reducing associated comorbidities such as lymphedema, and allowing to detect micro metastases after ultra-staging of the SLN.

Several studies have described the feasibility of the SLN technique in patients with early-stage EOC [12-24]. Most recent studies utilized a method in which a tracer is injected into the ovarian ligaments prior to tumor resection, with nearly all of these studies reporting very high detection rates $(71-100 \%)$ of at least one SLN. If intraoperative pathologic examination of the frozen section confirms an ovarian malignancy, lymph node resection is performed. If the result of the frozen section shows a benign or borderline tumor, staging is not required and tracer injection prior to resection would have been unnecessary. Unfortunately, pre-operative techniques to predict ovarian malignancy based on tumor markers and ultrasound (risk of malignancy index (RMI), International Ovarian Tumor Analysis (IOTA) models) are not performant enough to avoid surgery of benign adnexal masses [25, 26], resulting in needless injection of (radioactive) tracer in case of benign or borderline pathology.

The main objective of this study was to investigate whether the SLN mapping technique is applicable to patients who have already undergone ovarian tumor resection. Using this technique, the tracer is injected into the stumps of the ovarian ligaments if either the result of a frozen section confirms malignancy, or definitive pathology confirms malignancy and a second surgical procedure is performed.

\section{Materials and methods \\ Patients}

Patients who underwent laparotomy with frozen section for a pelvic mass suspicious for malignancy and those who underwent a second staging laparotomy after prior resection of a malignant ovarian mass at Maastricht University Medical Centre or Radboud University Medical Centre Nijmegen were included in this study. All patients provided fully informed written consent prior to study enrolment, and the study protocol was approved by the local ethics committee (approval number: NL53246.068.15). Patients were excluded if they had undergone previous vascular surgery of the aorta, caval vein, and/or iliac vessels; if they had undergone previous lymphadenectomy or lymph node sampling in the iliac or para-aortal region; if they had a history of malignant lymphoma or malignant tumor in the abdominal cavity; if they had experienced a previous allergic reaction to blue dye or human albumin; or if they were pregnant or lactating.

\section{SLN procedure}

Patients with high suspicion of a malignant ovarian tumor received general anesthesia. After median laparotomy, the ovarian tumor was removed and sent to the pathologist for frozen section analysis. In case of a benign or borderline result, SLN detection was not attempted. If the frozen section showed malignancy, four aliquots, each containing $0.2 \mathrm{ml}$ patent blue dye and $0.15 \mathrm{ml}$ $(20 \mathrm{MBq})$ of radioactive $99 \mathrm{mTc}$-nanocolloid (Nanocoll $^{\circledR}$; GE Healthcare, Eindhoven, the Netherlands), were injected into the dorsal and ventral sides of the remains of the ligamentum ovarium proprium and the ligamentum infundibulo-pelvicum (lateral side) just below the peritoneum (Fig. 1). After an interval of at least a $15 \mathrm{~min}$, the retroperitoneal space of the pelvic and para-aortic regions was opened, and the presence of $\operatorname{SLN}(\mathrm{s})$ was examined using the gamma-probe and/or visually (blue dye). The surgeon recorded the number and location of the resected SLNs. Twelve locations were assessed: the upper and lower para-aortic regions, the upper and lower inter-aortocaval regions, the upper and lower para caval regions, the right and left common iliac regions, the right and left external iliac regions, and the right and left obturator fossa regions. After removal of identified SLNs, the location was re-examined with the gamma probe for the presence of other lymph nodes containing more than $10 \%$ of the activity in the SLN. After this procedure with or without detection of SLNs, the complete standard staging procedure was performed, including a comprehensive random lymph node sampling of the bilateral pelvic and para aortal/caval region, hysterectomy, resection of the contralateral adnex and standard peritoneal biopsies.

The procedure performed in patients undergoing a second surgical staging procedure after resection of the ovarian malignancy was identical, starting with the injections of four aliquots of blue dye and radioactive tracer into the remnants of the ligaments.

\section{Histopathology}

SLNs and non-SLNs were examined separately. NonSLNs were cut into single sections and stained with hematoxylin and eosin ( $\mathrm{H} \& \mathrm{E})$, according to the standard protocol for lymph node examination. SLNs were cut into $2 \mathrm{~mm}$ sections for $\mathrm{H} \& \mathrm{E}$ staining. H\&E-negative SLNs in the first section were further cut into $0.5 \mathrm{~mm}$ sections and examined for the presence of micro metastases $(0.2 \mathrm{~mm})$. At each step, the sections were immunohistochemically stained with antibody to cytokeratin MNF-116. 


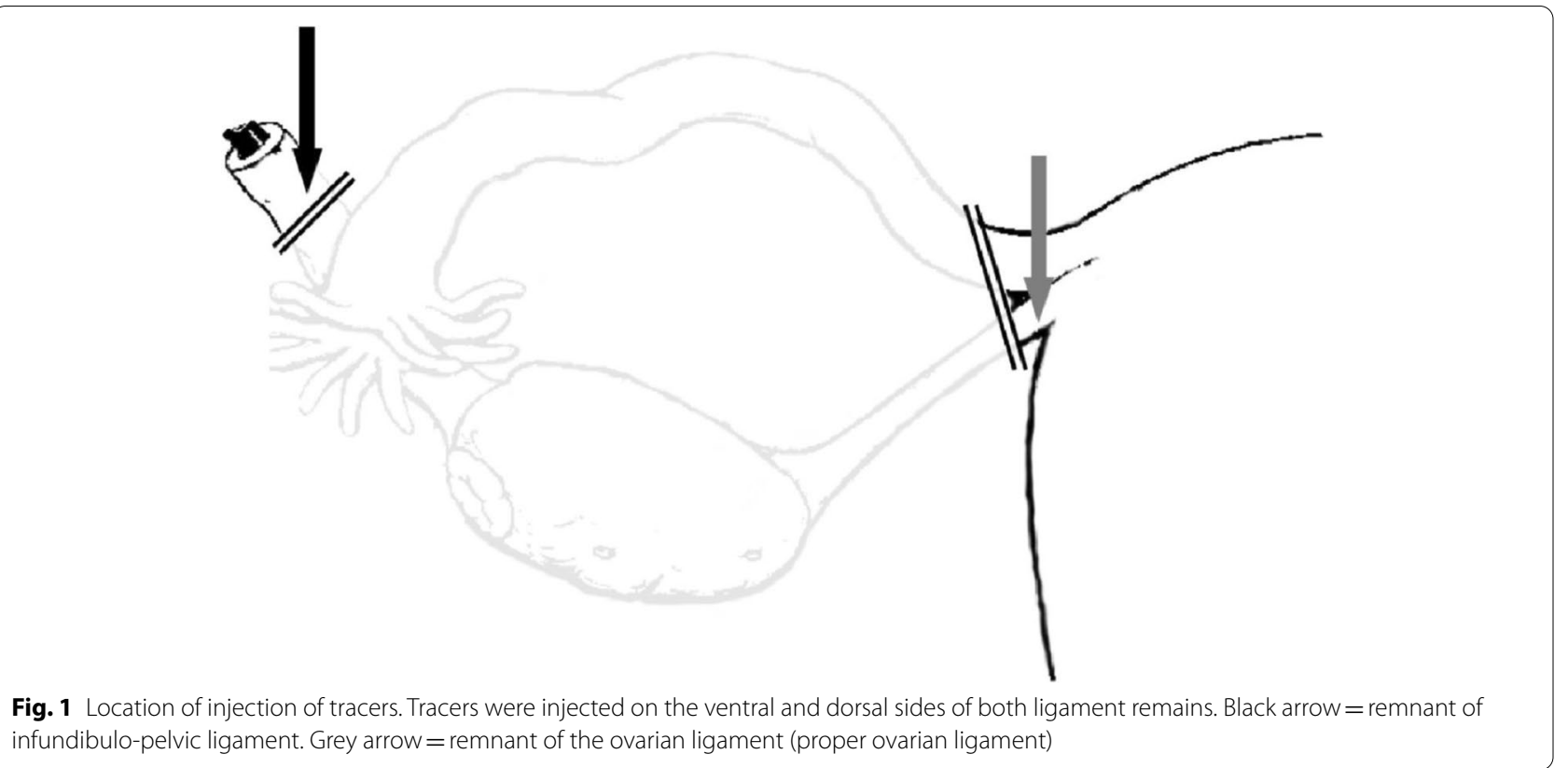

\section{Sample size calculation}

In a previous study, the rate of detection of hotspot(s) following injection of radioactive tracers and a $15 \mathrm{~min}$ waiting period before resecting the adnexal mass was $100 \%$ [15]. Based on these results, a sample of 20 evaluable patients was considered large enough to determine whether identification of SLNs is feasible (detection rate of at least 50\%) following a salpingo-oophorectomy and injection of tracers into the remnants of the ovarian ligaments. An interim analysis was planned after the evaluation of the first ten patients.

\section{Results}

The interim analysis was performed after the inclusion of eleven patients (the last two patients were included within 1 week). Patient characteristics are shown in Table 1. In three patients only, at least one SLN was identified (27, 95\%CI 10-54\%). Although the CI reached just above $50 \%$, this low percentage prompted us to terminate the study prematurely.

None of the patients experienced any allergic or adverse reactions. The SLN procedure was performed on the left side in six patients (54.4\%), on the right side in four patients $(36.4 \%)$, and on both sides in one patient (9.1\%). In eight patients, the SLN procedure was performed during the primary surgical procedure after the frozen section showed malignancy. In the remaining three patients, the SLN technique was performed during a second surgical procedure between 5 and 8 weeks after
Table 1 Patient characteristics

\begin{tabular}{ll}
\hline Number of patients & 11 \\
Median age & $57(44-79)$ \\
Pre- or post menopause & \\
- Post & $7(63,6 \%)$ \\
- Pre & $3(27,3 \%)$ \\
- Unknown & $1(9,1 \%)$ \\
Surgery & \\
- Frozen section & $8(72,7 \%)$ \\
- 2nd surgery & $3(27,3 \%)$ \\
Frozen section malignant & $8(100 \%)$ \\
FIGO Stage & $11(100 \%)$ \\
- 1 A & \\
- $1 B$ & $6(54,6 \%)$ \\
1C & 0 \\
2A & $4(36,3 \%)$ \\
Tumor type & 0 \\
- Clearcell & $1(9,1 \%)$ \\
Serous & \\
\hline Mucinous & $5(45,5 \%)$ \\
\hline
\end{tabular}

primary surgery. In all three cases where the SLN was successfully detected, the SLN technique was performed during primary surgery. 


\section{SLN detection and location after injection}

In three out of eleven patients SLN's were identified with the $\gamma$-probe $15 \mathrm{~min}$ after the injection of the radioactive tracer (Table 2). In two of these patients, one SLN each was identified on the ipsilateral side, the para-aortic, and the paracaval region. In the remaining patient, two SLNs were found, one in the inter-aorto-caval region and the other close to the common iliac artery on the contralateral side. No SLNs could be identified based on blue colorization. None of the SLNs were positive for metastasis. Furthermore, none of these three patients had lymph node metastases in the non-SLNs, which were resected to complete the staging procedure. Of the eight patients lacking SLN, one had a lymph node metastasis in a non-SLN.

\section{Discussion}

Many studies have evaluated the feasibility of detecting SLNs by injecting tracer(s) into the mesovarium and/or ovarian ligaments. Tracer injection prior to tumor resection and examination by frozen sections resulted in very high detection rates (87.5-100\%) of at least one SLN [12$22]$. The present study showed that injection of tracer into the remains of both ovarian ligaments after resection of the pathological adnex resulted in a low rate of SLN detection ( 3 out of 11 patients, $27 \%$; $95 \%$ CI $10-53 \%$ ). Too reach a detection rate of more than $50 \%$ as noted in the sample size calculation, we would have had to add at least 5 more patients with a positive node, which did not seem plausible in the current study design based on the results of the first 11 patients. For a detection rate comparable to the literature (90\%) [12-22] we would even have had to include 69 more patients with a positive node. Because of these results, the study was terminated prematurely and we concluded that the identification of the sentinel node is not efficient after prior resection of the adnexal mass.

Although the study population in our study was small, the SLN detection rate was disappointingly low. To date, only three (two of the same research group) other studies assessed the detection of SLNs following the injection of tracer into the remnants of the ovarian ligaments after the adnexa were resected, either during the same or a subsequent surgical procedure $[21,25,26]$.
In the study of Lago et al. the detection rate was $100 \%$ (10 out of 10 patients), being much higher than the $27 \%$ detection rate in the present study [21]. They injected the tracer deep into the parametrium rather than superficially under the peritoneum (shown on a video which was provided as an appendix), and also used indocyanine green as a tracer. The injection of the tracer deep into the parametrium resulted in a remarkably high number of cases with a pelvic (non-para aortal) sentinel node (88\%), while the three SLNs detected in our study were all located at the para-aortal/para-caval level. The same research group later published a phase II clinical trial named the SENTOV study enrolling 20 patients where again the number of pelvic sentinel lymph nodes was high (93\%) [24]. This may suggest that injection of the tracer after resection of the ovary deep into the parametrium, results in tracing uterine instead of ovarian pelvic sentinel lymph nodes. In this context, it should be noted that in a previous examaning ovarian lymphatic drainage we showed that the two major lymphatic drainage pathways of the ovaries invariably run via the suspensory ligament (infundibulopelvic ligament) and the proper ligament of the ovaries (ovarian ligament), the latter also being part of the uterine lymphatic drainage [27]. This would mean that with respect to the ovary, the presence of a pelvic sentinel node depends on whether the lymphatic flow direction in the ligament is from the ovary to the uterus or vice versa. Our lower para-aortal detection rate $(27 \%)$ when compared to Lago et al. (70 and 100\% respectively) may be the result of alteration of the lymphatic drainage after resection of the ovary, which makes detection of the actual para-aortal sentinel node after prior resection less reliable.

We do not think that the conflicting results can be explained by the use of different tracers. Indocyanine green is although its widespread use still not certified for its use as a tracer, and is particularly elegant for laparoscopy, which is in the setting of large adnexal masses warranting spill free resection not always possible. Furthermore, the advantage of ICG over radio-active technetium lies especially in the advantage of avoiding radio-activity, not in its detection performance since the effectiveness of technetium to detect the sentinel node is good, which was also the case in the SENTOV study (100\% detection with $37 \mathrm{MBq}$ radio-active technetium)

Table 2 Sentinel nodes fou

\begin{tabular}{|c|c|c|c|c|c|c|c|}
\hline Patient & Tumor side & $\begin{array}{l}\text { Number of } \\
\text { SLN }\end{array}$ & Histology during surgery & Location SLN & $\begin{array}{l}\text { Histology } \\
\text { after surgery }\end{array}$ & $\begin{array}{l}\text { Metastases in } \\
\text { the SLNs }\end{array}$ & $\begin{array}{l}\text { Metastases } \\
\text { in non-SLNs }\end{array}$ \\
\hline 1 & Right & 1 & At least borderline & Paracaval low right & Mucinous & No & No \\
\hline 2 & Left & 1 & Mixed & Para aortal low left & Clear cell & No & No \\
\hline 3 & Left & 2 & Clear cell & $\begin{array}{l}\text { Interaorta-caval, com- } \\
\text { mon iliac artery right }\end{array}$ & Clear cell & No & No \\
\hline
\end{tabular}


and our previous research where good detection rates were reached with the same dose $(20 \mathrm{MBq})$ radio-active technetium injected prior to resection of the adnex [15].

The third study examining the detection of SLNs following the injection of tracer into the remnants of the ovarian ligaments after the adnexa were resected, is a preliminary analysis of the first 31 patients enrolled in the SELLY trial, in 13 patients a secondary staging procedure was performed after an incidental diagnosis of ovarian cancer [23]. Using ICG as a SLN tracer they also reached a detection rate of $41,7 \%$ after resection of the adnexal mass, compared to $88,9 \%$ when immediate staging was performed. These results support our conclusion that the best results in identifying SLNs are obtained when the tracer is injected before the pathological ovary is resected.

The research group of Lago recently published a protocol for ultrastaging of the sentinel node, using section levels deepening every $200 \mu \mathrm{m}$ - which is more detailed than the section levels every $500 \mu \mathrm{m}$ used in the current study. The described technique allows an even more reliable detection of micro metastases [28].

The differences in injection tracers, doses and histopathological protocol used between the different studies urge the need for uniform standardization of the sentinel node technique in ovarian cancer, before it can successfully implemented in routine clinical practice.

\section{Conclusion}

The SLN mapping technique has shown high detection rates of $87.5-100 \%$ when tracers are injected before resecting the adnexa [12-22]. This technique therefore seems feasible and may prevent lymphadenectomy. The present study showed that the rate of SLN detection after prior tumor resection was disappointingly low (27\%). Injection of (radioactive) tracer is therefore not accurate to detect the sentinel node when after resection of the adnex, frozen sections show malignancy.

Based on these results, we support a standardized technique as proposed previously by Dell'Orto et al. [23], of one injection in both the suspensory and the infundibulopelvic ligament of the ovary in all patients with a high suspicion of an ovarian mass limited to the ovary. The use of indocyanine green as an alternative tracer for radio-active technetium seems promising but still needs to be examined on a larger scale especially in the case of laparotomy since its use for laparotomic staging of ovarian cancer has been reported in only a limited number of cases. Recognizing that a number of centers will not have near-infrared fluorescent technology, the use of radiocolloid and blue dye is an acceptable alternative. After a waiting time of $10-15 \mathrm{~min}$ after injection, the suspicious ovarian mass should be removed for frozen section analysis. If frozen section analysis confirms a malignancy, the staging procedure can start, including removal of the identified SLNs.

Using the proposed standardized technique, a protocol is designed to examine the accuracy and benefits of the SLN technique in an international multi-center collaborative study; based on the injection of tracers in the ovarian ligaments in patients with clinical early-stage ovarian cancer.

\section{Abbreviations \\ SLN: Sentinel lymph node; EOC: Early stage ovarian cancer.}

\section{Acknowledgments}

Not applicable.

\section{Authors' contributions}

$\mathrm{PL}$ wrote manuscript and collected data. RK and SL were principal investigators in Maastricht. PZ was principal investigator in Nijmegen. BS and TVG helped collecting patients for the study. JVP provided knowledge and helped with the radioactive tracers during surgery. All authors read and approved the final manuscript.

\section{Funding}

No funding was to be declared.

\section{Availability of data and materials}

The datasets used and/or analysed during the current study are available from the corresponding author on reasonable request.

\section{Declarations}

\section{Ethics approval and consent to participate}

All patients provided fully informed written consent prior to study enrolment, and the study protocol was approved by the local ethics committee at Maastricht university medical centre (approval number: NL53246.068.15).

\section{Consent for publication}

All authors consent for publication.

\section{Competing interests}

The authors declare that they have no competing interests.

\section{Author details}

${ }^{1}$ Department of Obstetrics and Gynecology, Maastricht University Medical Center, Maastricht, The Netherlands. ${ }^{2} \mathrm{GROW}$ - School for Oncology and Developmental Biology, Maastricht University Medical Center, Maastricht, The Netherlands. ${ }^{3}$ Department of Obstetrics and Gynecology, Radboud University Medical Centre, Nijmegen, The Netherlands. ${ }^{4}$ Present address: Department of Obstetrics and Gynecology, Leuven University Medical Centre, Leuven, Belgium. ${ }^{5}$ Department of Radiology Nuclear Medicine, Maastricht University Medical Center, Maastricht, The Netherlands.

Received: 23 April 2020 Accepted: 27 September 2021

Published online: 13 October 2021

References

1. Kleppe M, Wang T, Van Gorp T, Slangen BF, Kruse AJ, Kruitwagen RF. Lymph node metastasis in stages I and II ovarian cancer: a review. Gynecol Oncol. 2011;123:610-4.

2. Maggioni A, Benedetti Panici P, Dell'Anna T, et al. Randomised study of systematic lymphadenectomy in patients with epithelial ovarian cancer macroscopically confined to the pelvis. Br J Cancer. 2006;95:699Y704.

3. Harter P, Gnauert K, Hils R, et al. Pattern and clinical predictors of lymph node metastases in epithelial ovarian cancer. Int J Gynecol Cancer. 2007;17:1238Y1244 
4. Ditto A, Martinelli F, Reato C, et al. Systematic Para-aortic and pelvic lymphadenectomy in early stage epithelial ovarian cancer: a prospective study. Ann Surg Oncol. 2012;19:3849Y3855.

5. Carnino F, Fuda G, Ciccone G, et al. Significance of lymph node sampling in epithelial carcinoma of the ovary. Gynecol Oncol. 1997;65:467Y472.

6. Benedet JL, Bender H, Jones H III, Ngan HY, Pecorelli S. FIGO staging classifications and clinical practice guidelines in the management of gynecologic cancers. FIGO committee on gynecologic oncology. Int J Gynaecol Obstet. 2000;70:209-62.

7. Angioli R, Plotti F, Palaia I, et al. Update on lymphadenectomy in early and advanced ovarian cancer. Curr Opin Obstet Gynecol. 2008;20:34-9.

8. Di Re F, Baiocchi G. Value of lymph node assessment in ovarian cancer: status of the art at the end of the second millennium. Int J Gynecol Cancer. 2000;10:435-42.

9. Gipponi M, Bassetti C, Canavese G, Catturich A, Di Somma C, Vecchio C, et al. Sentinel lymph node as a new marker for therapeutic planning in breast cancer patients. J Surg Oncol. 2004;85(3):102-11.

10. Landi G, Polverelli M, Moscatelli G, Morelli R, Landi C, Fiscelli O, et al. Sentinel lymph node biopsy in patients with primary cutaneous melanoma: study of 455 cases. J Eur Acad Dermatol Venereol. 2000;14(1):35-45.

11. Levenback CF, Ali S, Coleman RL, Gold MA, Fowler JM, Judson PL, et al. Lymphatic mapping and sentinel lymph node biopsy in women with squamous cell carcinoma of the vulva: a gynecologic oncology group study. J Clin Oncol. 2012;30(31):3786.

12. Vanneuville G, Mestas D, Le Bouedec G, et al. The lymphatic drainage of the human ovary in vivo investigated by isotopic lymphography before and after the menopause. Surg Radiol Anat. 1991;13(3):221-6.

13. Negishi $H$, Takeda M, Fujimoto T, et al. Lymphatic mapping and sentinel node identification as related to the primary sites of lymph node metastasis in early stage ovarian cancer. Gynecol Oncol. 2004;94(1):161-6.

14. Nyberg RH, Korkola P, Mäenpää J. Ovarian sentinel node: is it feasible? Int J Gynecol Cancer. 2011;21(3):568-72.

15. Kleppe M, Brans B, van Gorp T, et al. The detection of sentinel nodes in ovarian cancer: a feasibility study. J Nucl Med. 2014;55:1799Y1804.

16. Angelucci M, Corrado G, Vizza E. Laparoscopic indocyanine green sentinel lymph node mapping in early ovarian cancer. A pilot study and review of the literature. Ital J Gynaecol Obstet. 2016;28:N. 5 Quarterly - ISSN 2385-0868.

17. Hassanzadeh M, Farahabadi EH, et al. Lymphatic mapping and sentinel node biopsy in ovarian tumors: a study using intra- operative Tc-99m-Phytate and lymphoscintigraphy imaging. J Ovarian Res. 2016;9(1):55.
18. Nyberg RH, Korkola P, Mäenpää JU. Sentinel node and ovarian tumors: a series of 20 patients. Int J Gynecol Cancer. 2017;27(4):684-9.

19. Buda A, Passoni P, Corrado G, et al. Near-infrared fluorescence-guided sentinel node mapping of the ovary with indocyanine green in a minimally invasive setting: a feasible study. J Minim Invasive Gynecol. 2017;24(1):165-70.

20. Speth SC, Kruitwagen RF, Kleppe M, et al. Comparison of intraoperative $\gamma$-probe imaging and postoperative SPECT/CT in detection of sentinel nodes related to the ovary. J Nucl Med. 2017;58(2):243-5.

21. Lago V, Bello P, Montero B, et al. Clinical application of the sentinel lymph node technique in early ovarian cancer: a pilot study. Int J Gynecol Cancer. 2019;29(2):377-81.

22. Dell'Orto F, Laven P, Delle Marchette M, Lambrechts S, Kruitwagen R, Buda A. Feasibility of sentinel lymph node mapping of the ovary: a systematic review. Int J Gynecol Cancer. 2019;29:1209-15.

23. Ucella S, Nero C, Vizza E, Vargiu V, Corrado G. Sentinel-node biopsy in early-stage ovarian cancer: preliminary results of a prospective multicentre study (SELLY). Am J Obstet Gynecol. 2019;221(4):324.e1-324.e10.

24. Lago V, Bello P, Montero B, Matute L, Padilla-Iserte P. Sentinel lymph node technique in early-stage ovarian cancer (SENTOV): a phase II clinical trial. Int J Gynecol Cancer. 2020 Sep;30(9):1390-6.

25. Ratnavelu NDG, Brown AP, Malllett S, Scholten RJPM. Intraoperative frozen section analysis for the diagnosis of early stage ovarian cancer in suspicious pelvic masses intraoperative frozen section analysis for the diagnosis of early stage ovarian cancer in suspicious pelvic masses. Cochrane Database Syst Rev. 2016;3(3):CD010360.

26. Meys EMJ, Kaijser J, Kruitwagen RFPM, Slangen BFM, Van Calster B, Aertgeerts B, et al. Subjective assessment versus ultrasound models to diagnose ovarian cancer: a systematic review and meta-analysis. Eur J Cancer. 2016;58:17-29.

27. Kleppe M, Kraima AC, Kruitwagen RF, Van Gorp T, Smit NN, van Munsteren $J C$, et al. Understanding lymphatic drainage pathways of the ovaries to predict sites for sentinel nodes in ovarian cancer. Int J Gynecol Cancer. 2015;25:1405-14

28. Lago V, Montero B, López S, Padilla-Iserte P, Matute L, Marina T, et al. Ultrastaging protocol in sentinel lymph node for apparent early stage ovarian cancer. Gynecol Oncol. 2021;161:408-13.

\section{Publisher's Note}

Springer Nature remains neutral with regard to jurisdictional claims in published maps and institutional affiliations.
Ready to submit your research? Choose BMC and benefit from:

- fast, convenient online submission

- thorough peer review by experienced researchers in your field

- rapid publication on acceptance

- support for research data, including large and complex data types

- gold Open Access which fosters wider collaboration and increased citations

- maximum visibility for your research: over $100 \mathrm{M}$ website views per year

At BMC, research is always in progress.

Learn more biomedcentral.com/submissions 